\title{
HRP-2 determines HIV-1 integration site selection in LEDGF/p75 depleted cells
}

\author{
Rik Schrijvers' ${ }^{1}$, Sofie Vets ${ }^{1}$, Jan De Rijck' ${ }^{1}$ Nirav Malani ${ }^{2}$, Frederic D Bushman² ${ }^{2}$ Zeger Debyser ${ }^{1}$ and Rik Gijsbers ${ }^{* *}$
}

\begin{abstract}
Background: Lens epithelium-derived growth factor (LEDGF/P75) is a cellular co-factor of HIV-1 integrase (IN) that tethers the viral pre-integration complex to the host cell chromatin and determines the genome wide integration site distribution pattern of HIV-1. Recently, we demonstrated that HIV-1 replication was reduced in LEDGF/p75 knockout (KO) cells. LEDGF/p75 KO significantly altered the integration site preference of HIV-1, but the pattern remained distinct from a computationally generated matched random control set (MRC), suggesting the presence of an alternative tethering factor. We previously identified Hepatoma-derived growth factor related protein 2 (HRP-2) as a factor mediating LEDGF/p75-independent HIV-1 replication. However, the role of HRP-2 in HIV-1 integration site selection was not addressed.

Findings: We studied the HIV-1 integration site distribution in the presence and absence of LEDGF/p75 and/or HRP-2, and in LEDGF/p75-depleted cells that overexpress HRP-2. We show that HRP-2 functions as a co-factor of HIV-1 IN in LEDGF/p75-depleted cells. Endogenous HRP-2 only weakly supported HIV-1 replication in LEDGF/p75 depleted cells. However, HRP-2 overexpression rescued HIV-1 replication and restored integration in RefSeq genes to wild-type levels. Additional HRP-2 KD in LEDGF/p75-depleted cells reduces integration frequency in transcription units and shifts the integration distribution towards random.

Conclusions: We demonstrate that HRP-2 overexpression can compensate for the absence of LEDGF/p75 and indicate that the residual bias in integration targeting observed in the absence of LEDGF/p75 can be ascribed to HRP-2. Knockdown of HRP-2 upon LEDGF/p75 depletion results in a more random HIV-1 integration pattern. These data therefore reinforce the understanding that LEDGF/p75 is the dominant HIV-1 IN co-factor.
\end{abstract}

Keywords: LEDGF/p75, HRP-2, HIV-1, Targeting, Integration site analysis

\section{Findings}

HIV-1 integration target site selection is not a random event, but preferentially occurs in the body of active transcription units [1], due to the interaction with the cellular co-factor LEDGF/p75 [2,3]. Knockdown (KD) or $\mathrm{KO}$ of LEDGF/p75 shifts HIV-1 integration targeting away from transcription units [2-7]. LEDGF/p75 functions as a molecular tether, interacting with the viral integrase (IN) via its C-terminal integrase binding domain (IBD) $[8,9]$, and with the host-cell chromatin via its $\mathrm{N}$-terminus. The latter contains chromatin-binding motifs such as the PWWP-domain, AT-hook-like motifs, and a set of charged regions (Figure 1) $[6,10]$. Several

\footnotetext{
*Correspondence: rik.gijsbers@med.kuleuven.be

${ }^{1}$ Division of Molecular Medicine, Katholieke Universiteit Leuven, Leuven, Flanders, Belgium

Full list of author information is available at the end of the article
}

lines of evidence support this tethering and targeting model for LEDGF/p75 in HIV-1 replication. The chromatin binding sites of LEDGF/p75 correlate with the HIV-1 integration distribution pattern [11], and LEDGF/ p75-hybrids in which the N-terminus is replaced by an alternative chromatin interaction domain, such as the heterochromatin binding element CBX1, have been shown to retarget HIV-1 integration out of transcription units and towards heterochromatic regions $[5,12]$.

We reported that LEDGF/p75 KO shifts HIV-1 integration away from transcription units [7], consistent with previous LEDGF/p75 KD experiments [2,3]. However, even in the absence of LEDGF/p75, HIV-1 significantly favored integration in transcription units when compared to random [7]. This may be explained by an intrinsic feature of the IN protein or the pre-integration complex, or by the presence of one or more additional 


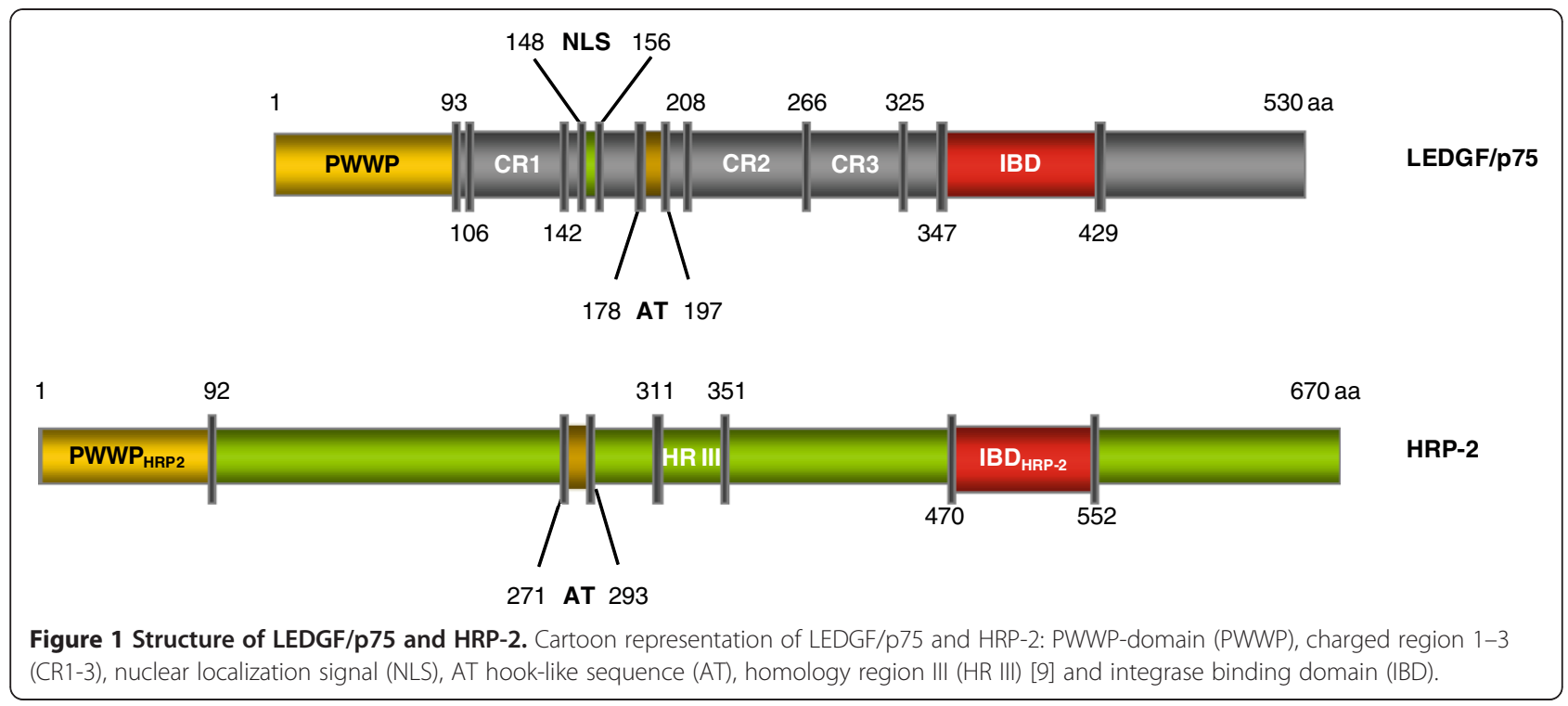

co-factors that target integration into transcription units. Recently, we reported that HRP-2, the only known cellular protein besides LEDGF/p75 that combines a PWWPdomain and an IBD-like domain [9,13] (Figure 1), plays a role in LEDGF/p75-independent HIV-1 replication in both human LEDGF/p75 KO and KD cell lines [7]. In this study we examined the contribution of HRP-2 in directing integration site selection of HIV-1.

We and others have demonstrated that backcomplementation (BC) of LEDGF/p75 in LEDGF/p75depleted cells rescued HIV-1 replication and restored the integration site distribution to wild-type (wild-type) patterns [5,14]. Here, we first assessed the potential of HRP-2 to complement LEDGF/p75-depleted cells. Overexpression of HRP-2 (9-fold compared to endogenous wild-type levels, Figure 2A) in LEDGF/p75-depleted cells could substitute in part for LEDGF/p75. Infection with single-round HIV-fLuc virus was restored to $62 \pm 8 \%$ compared to LEDGF/p75 BC cells (Figure 2B, compare LEDGF/p75 BC and LEDGF/p75 KD + HRP-2). Replication of HIV-1 $1_{\mathrm{NL} 4.3}$ virus was rescued to near wild-type levels upon HRP-2 overexpression as seen with LEDGF/ p75 BC (Figure 2C). Both LEDGF/p75 and HRP-2 mediated rescue correlated with an increase in integrated proviral copies (Figure 2D). Next, in line with previous observations [15], the nuclear distribution pattern of LEDGF/p75 during interphase was speckled, while that of HRP-2 was homogenous (Additional file 1, compare row 2 and 3). Unlike LEDGF/p75, HRP-2 did not bind mitotic chromosomes (Additional file 1, compare row 5 and 6), although this might not be relevant for HIV-1 replication since LEDGF/p75 depletion in non-dividing macrophages also affects HIV-1 replication [16]. Still, HRP-2 overexpression relocated IN to the nucleus in LEDGF/p75 KD cells (Additional file 1, row 3 ), suggesting a direct interaction.

Previously, we demonstrated that HRP-2 KD in LEDGF/p75-depleted cells additionally hampered HIV-1 replication (see reference [7] and data not shown). Here, we evaluated the effect of additional HRP-2 KD in LEDGF/p75-depleted cells on HIV-1 integration site distribution. We generated stable HRP-2 KD and LEDGF/ p75 KD cells $(86 \%$ and $>90 \%$ KD on mRNA level for HRP-2 and LEDGF/p75, respectively), double KD cells (>90\% LEDGF/p75 KD and 84\% HRP-2 KD on mRNA level) and complemented LEDGF/p75 KD cells with HRP-2 (Figure 2A and data not shown). The respective cell lines were challenged with HIV-fLuc integration sites were amplified and their distribution pattern was characterized.

First, we generated a genomic heat map describing the integration site distribution for a subset of genomic features (Additional file 2). The color of each tile represents the deviation from random $(\mathrm{MRC}=0.5)$ for the examined feature, ranging from red (favored compared to $\mathrm{MRC}$ ) over white to blue (disfavored compared to MRC). While tile colors did not differ when HRP-2 was depleted in wild-type cells (compare wild-type and HRP-2 KD), a shift in tile color towards random could be appreciated upon LEDGF/p75 KD (compare wildtype and LEDGF/p75 KD), which was even more pronounced upon the additional suppression of HRP-2 expression (compare LEDGF/p75 KD and LEDGF/p75 KD HRP-2 KD). More detailed analysis showed that integration in RefSeq genes was favored in wild-type cells ( $77.5 \%$ in genes, $\mathrm{p}<0.001$ compared to $\mathrm{MRC}$ ) and decreased significantly upon LEDGF/p75 KD (67.7\% in genes, $\mathrm{p}<0.001$ compared to $\mathrm{MRC}$ ), consistent with 
A
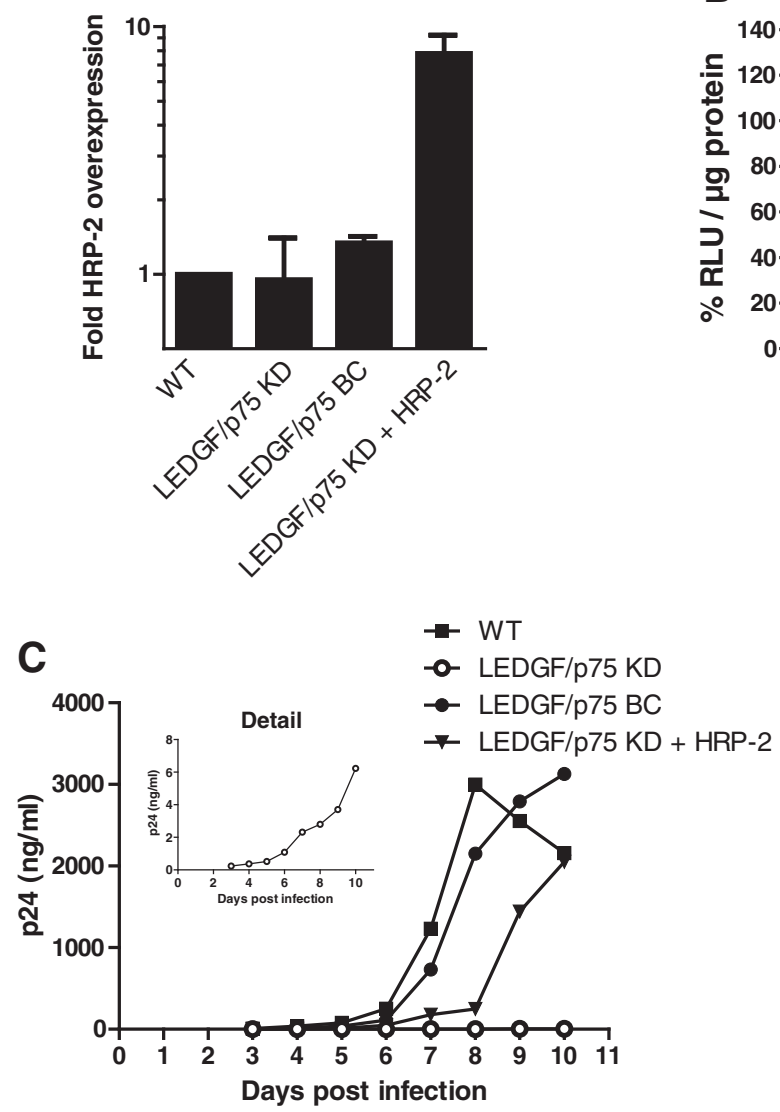

B

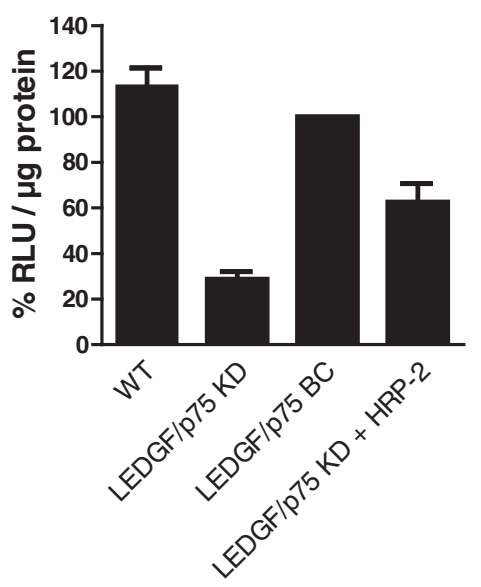

D

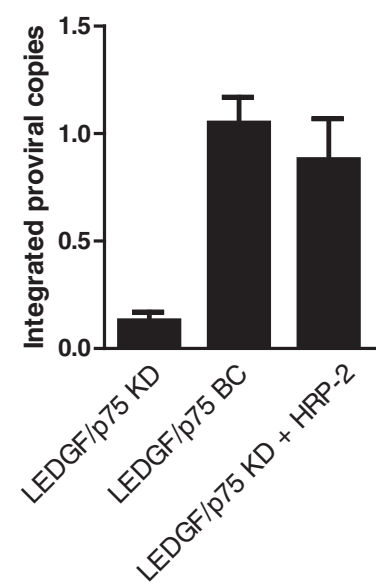

Figure 2 HRP-2 overexpression rescues HIV-1 replication. WT and stable LEDGF/p75 KD cell lines (LEDGF/p75 KD) were complemented with LEDGF/p75 (LEDGF/p75 BC) or HRP-2 (LEDGF/p75 KD + HRP-2). (A) HRP-2 mRNA expression levels shown as fold overexpression compared to WT. (B) Relative luciferase activity (RLU/ug protein) following HIV-fLuc transduction. Data were compiled from at least six independent experiments and expressed as percentages relative to LEDGF/p75 BC (mean \pm SD). (C) Multiple round HIV-1 replication after challenging the indicated cell lines with the laboratory strain HIV-1 ${ }_{N L 4.3}$. Replication was monitored by measuring the p24 content in the supernatant until day 10 at which point cells were confluent or showed massive cell death due to CPE. P24 levels decreased after day 8 in the WT condition due to CPE. Experiments were performed at least three times, a representative experiment is shown. (D) At day 10 of the experiment shown in (C), cells were split and maintained under antiretroviral therapy $\left(A Z T, 50 \times \mid C_{50}\right)$ for 10 days to dilute all non-integrated viral DNA forms, before determining the number of integrated copies using qPCR.

previous results [2,5,6] (Table 1, Figure 3). HRP-2 overexpression in LEDGF/p75 KD cells rescued proviral integration in transcription units $(77.5 \%$, no difference compared to wild-type; Table 1, Figure 3 and Additional file 2). KD of HRP-2 in wild-type cells did not affect integration site distribution $(80.2 \%$ in genes, $\mathrm{p}=0.3$ for the comparison of wild-type and HRP-2 KD), confirming the dominant role of LEDGF/p75 over HRP-2. However, KD of HRP-2 in LEDGF/p75-depleted cells resulted in an additional decrease of integration in RefSeq genes, shifting integration out of transcription units and towards random (67.7\% in LEDGF/p75 KD versus $53.7 \%$ in LEDGF/p75 KD HRP-2 KD, p<0.001) (Table 1, Figure 3). Together these data provide evidence for a role of HRP-2 in targeting HIV-1 integration in LEDGF/ p75-depleted cells. Using a panel of histone modifications we also evaluated the frequency of integration near transcriptionally repressed regions (either silent regions, e.g. H3K27me3, or heterochromatin, e.g. H3K 9me3, H3K79me3, H4K20me3), as well as near marks associated with activation (e.g. H2BK5me1, H3K9me1, and H4K20me1). Since only a limited number of marks have been mapped in HeLa cells [17], we also included marks that were defined in $\mathrm{CD}^{+}$T-cells 
Table 1 Integration frequency of HIV in RefSeq genes

\begin{tabular}{llcc}
\hline & Cell line & \# sites & \% in RefSeq genes \\
\hline HIV-fLuc & WT & 1468 & $77.5^{* * \mid}|n|^{* * *}$ \\
sites & HRP-2 KD & 359 & $80.2^{* * *|n s|^{* * *}}$ \\
& LEDGF/p75 KD & 477 & $67.7^{* * * * * * \mid n s}$ \\
& LEDGF/p75 KD HRP-2 KD & 676 & $53.7^{* * *|* * *| * * *}$ \\
& LEDGF/p75 KD + HRP-2 & 445 & $77.5^{\left.* * *|n s|\right|^{* * *}}$ \\
MRC sites & WT & 4404 & 39.7 \\
(HIV-fLuc) & HRP-2 KD & 1077 & 37.9 \\
& LEDGF/p75 KD & 1431 & 40.3 \\
& LEDGF/p75 KD HRP-2 KD & 2028 & 39.3 \\
& LEDGF/p75 KD + HRP-2 & 1335 & 40.2 \\
\hline
\end{tabular}

Abbreviations: MRC matched random control, ns non-significant ( $\mathrm{p} \geq 0.05)$. Asterisks represent $p$-values $\left({ }^{*} p<0.05,{ }^{* *} p<0.01,{ }^{* * *} p<0.001\right.$ ) given after comparison with MRC|WT|LEDGF/p75 KD respectively. Significance was determined using a two-tailed Chi-square test. P-values were not corrected for multiple comparisons; alpha level is 0.003 after Bonferroni-correction (0.05/15).

[18] (Figure 4). HIV-1 integration in wild-type cells is generally disfavored near marks associated with transcriptionally silent regions and heterochromatin. LEDGF/p75 depletion shifts this phenotype more to MRC. In line with our previous data, additional HRP-2 KD in LEDGF/p75-depleted cells shifted integration distribution more towards MRC, indicating that the integration is more random upon double $\mathrm{KD}$ (Figure 4, compare LEDGF/p75 KD and LEDGF/p75 KD HRP-2 KD). Likewise, integration near histone marks associated with active transcription is reduced upon LEDGF/p75 KD and even more upon additional HRP-2 KD. HRP-2 overexpression restored the integration pattern near histone marks to levels observed in wild-type cells. The possible effects of such altered integration site distribution on proviral gene expression remain to be investigated. Since LEDGINs [19], allosteric IN inhibitors targeting the LEDGF/p75 binding site in IN, interfere with the interaction of either LEDGF/p75 or HRP-2 [7], LEDGINs might affect HIV-1 integration site distribution. Comparable data were obtained when transducing the same cell lines with an HIV-derived lentiviral vector (Additional file 3; Table S1).

Taken together, our work underscores the dominant role of LEDGF/p75 over HRP-2 in HIV-1 replication, which can be explained by the lower affinity of HRP-2 for HIV-1 IN compared to LEDGF/p75 [7,9,15]. Since HRP-2 overexpression in LEDGF/p75-depleted cells rescues HIV-1 replication, the relative expression levels of LEDGF/p75 and HRP-2 in relevant primary host cells are of importance. Different groups have reported on expression levels of LEDGF/p75 in primary activated and resting T-cells [21] or in different patient populations $[22,23]$, yet the levels in other subsets of HIV target-cells as well as the HRP-2 expression levels remain to be

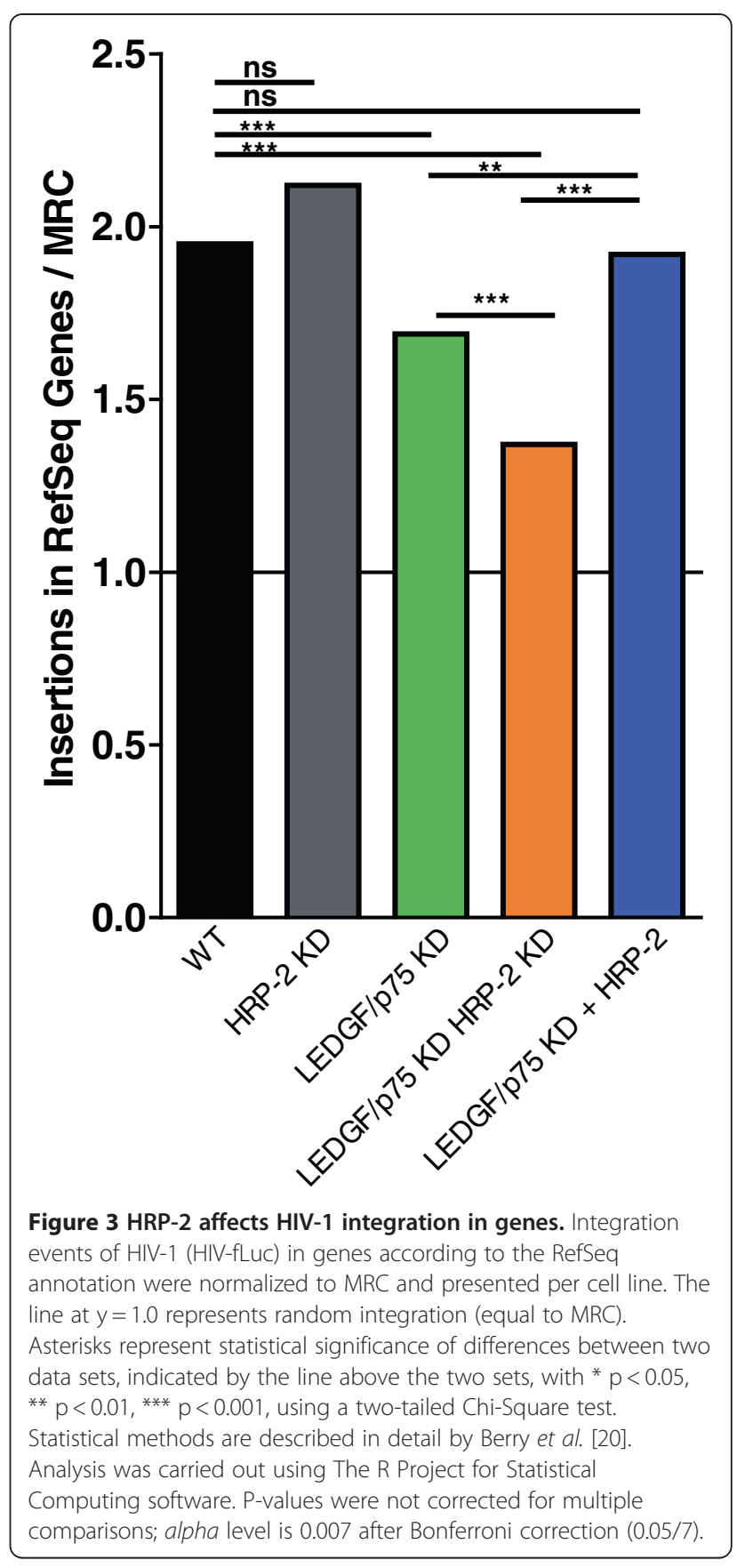

determined. Currently, there is no evidence that HRP-2 plays a role in HIV-1 replication in cells expressing WT levels of LEDGF/p75. Both LEDGF/p75 and HRP-2 carry a PWWP domain, recently identified as a chromatin reader recognizing epigenetic marks, such as methylated histone side-chains [6,24,25]. Previously, we demonstrated that swapping the PWWP-domain of LEDGF/ p75 with that of HRP-2 could rescue lentiviral replication and integration site selection in genes [6]. Here, integration distribution in LEDGF/p75 KD cells overexpressing HRP-2 was comparable to wild-type cells 


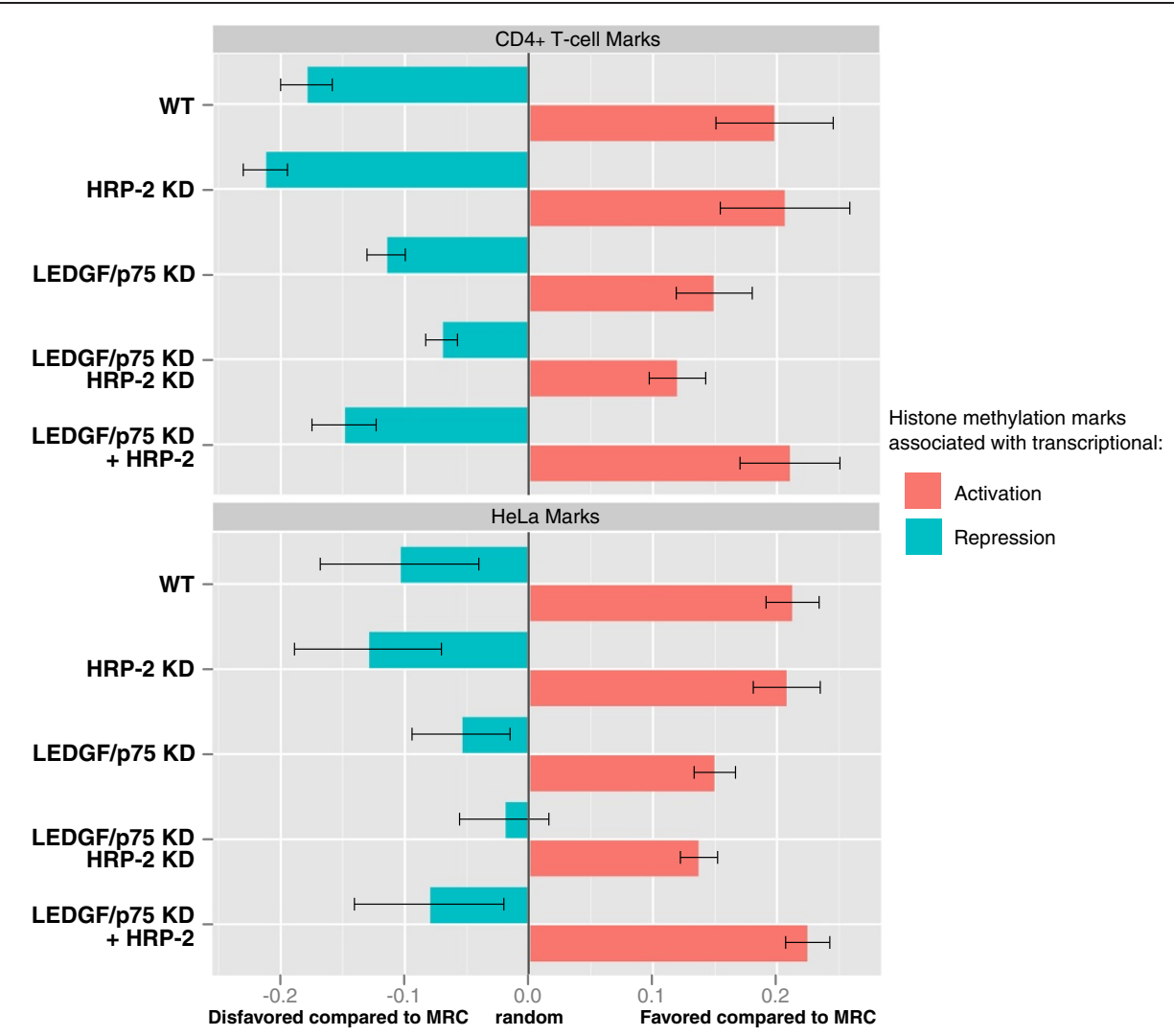

Figure 4 LEDGF/p75 and HRP-2 depletion shifts integration towards random. A pooled score was determined for HIV-1 integration near epigenetic marks associated with transcriptional repression or activation for each cell line and plotted in a bar diagram. The X-axis represents deviation from random with positive values indicating favored integration compared to MRC, negative values disfavored integration compared to MRC. The pooled score is the average of the different ROC values - 0.5 (to indicate the deviation from random) obtained with individual markers (not shown) in a $10 \mathrm{~kb}$ window. For HeLaP4, H3K9me2, H3K9me3, H3K27me3, and H4K20me3 were pooled as associated with 'repression'; H2BK5me1, H3K4me1, H3K36me3, H4K20me1 were pooled as associated with 'activation'. For CD4 ${ }^{+}$T-cells, H3K9me2, H3K9me3, H3K27me2, H3K27me3, H3K79me3 and H4K2Ome3 were pooled as associated with 'repression'; H2BK5me1, H3K4me1, H3K4me2, H3K9me1, H3K27me1, H3K36me3, H4K20me1 were pooled as associated with 'activation'.

(Figure 3 and Additional file 2 and 3), suggesting only subtle differences between LEDGF/p75 and HRP-2 for the interaction with the local chromatin. Although LEDGF/p75 and HRP-2 double KD shifted integration significantly out of transcription units, integration remained distinct from random in the double KD cells (Table 1). Multiple (mutually non-exclusive) reasons can be put forward to explain this observation. First, in these experiments we employed RNAi and even though the knockdown was potent $(>90 \%$ and $84 \%$ on mRNA level for LEDGF/p75 and HRP-2 respectively), residual LEDGF/p75 and HRP-2 might account for the residual bias. The generation of a human double KO cell line, devoid of both LEDGF/p75 and HRP-2, will provide a more definite answer. Second, this result could suggest the presence of additional cellular co-factor(s) that affect targeting in the absence of LEDGF/p75 and HRP-2. Transcription factor IIS (TFIIS) for example harbors an IBD-like domain [6,8], although structurally more distantly related to the IBD of LEDGF/p75 and HRP-2, but lacks a PWWP-domain. However, in the presence of LEDGF/p75, HRP-2 does not seem to play a role in HIV replication [6] or targeting (this work), suggesting alternate IBD containing tethers will probably only play a minor role in HIV replication or targeting in WT conditions, unless expression levels differ strongly, accrediting LEDGF/p75 as principal tether [6]. Third, the bias might reflect specific constraints of the viral IN, the local chromatin environment or the pre-integration complex itself. Although HIV integration favors weak palindromic sequences, our analysis indicated that this preference is irrespective of the presence or absence of LEDGF/p75 and/or HRP-2 ([6] and data not shown).

In conclusion, our data provide an explanation for why LEDGF/p75 depletion alone does not completely retarget integration distribution towards random, and they fit with previous data that HRP-2 binds the IN dimer with lower affinity $[7,9]$. These data also reinforce 
our understanding that LEDGF/p75 is the dominant cellular co-factor determining HIV-1 integration site selection.

\section{Additional files}

Additional file 1: HRP-2 overexpression relocates integrase to the nucleus of LEDGF/p75 depleted cells. Cells were transfected with plasmid encoding mRFP-IN and laser scanning microscopy images of cells stained with anti-LEDGF/p75 (LEDGF/p75) or anti-Flag (HRP-2) antibody are shown (green). Nuclei were stained with DAPI (4',6diamidino-2-phenylindole; blue). The respective constructs and cell lines are indicated. Interphase and mitotic cells are displayed separately. The data are representative for the vast majority of the imaged cells.

\section{Additional file 2: Heat map of integration frequency relative to} genomic features. Heat maps were developed to summarize relationships of proviral integration sites with genomic features using the receiver operating characteristic (ROC) area method [20]. The analyzed genomic features are mentioned on the left of the corresponding row of the heat map. Tile color indicates whether a chosen feature is favored (red, enrichment compared with random) or disfavored (blue, depletion compared with random) for integration for the respective data sets relative to their MRCs, as detailed in the colored $\mathrm{ROC}$ area scale at the bottom of the panel. The different data sets used are indicated above the columns. The asterisks denote significant differences of HIV integration compared to the LEDGF/p75 KD cell line for the respective features $\left({ }^{*}, p<0.05,{ }^{* *}, p<0.01 ;{ }^{* * *}, p<0.001\right.$, using Wald statistics referred to a Chi-square distribution), dashes overlay control tiles. The naming of the genomic features is described in Berry et al. [20]; TSS, transcription start site.

Additional file 3: Table S1. Integration frequency of HIV-derived lentiviral vector in RefSeq genes.

\section{Competing interests}

The authors declare no conflict of interest.

\section{Authors' contributions}

RS, SV, JDR, ZD, and RG conceived and designed the experiments. RS, SV, $J D R, R G$ performed the experiments. RS, NM, FDB, ZD, RG analyzed the data. $\mathrm{RS}, \mathrm{RG}$ wrote the paper. All authors read and approved the final manuscript.

\section{Funding}

RS and JDR are doctoral fellows of the Flemish Fund for Scientific Research (FWO Vlaanderen), SV is a doctoral fellow of the Iwild-type. JDR is holder of a Mathilde-Krim postdoctoral fellowship (amfAR). Research was funded by grants from the Iwild-type (SBO grant CellCoVir), the FWO and the EU (FP7 THINC). This work was supported by NIH grants Al52845 and Al082020, the University of Pennsylvania Center for AIDS Research, and the Penn Genome Frontiers Institute with a grant with the Pennsylvania Department of Health. The funders had no role in study design, data collection and analysis, decision to publish, or preparation of the manuscript.

\section{Acknowledgements}

We thank Paulien Van de Velde and Barbara Van Remoortel for excellent technical assistance.

\section{Author details}

'Division of Molecular Medicine, Katholieke Universiteit Leuven, Leuven, Flanders, Belgium. ${ }^{2}$ Department of Microbiology, University of Pennsylvania School of Medicine, Philadelphia, Pennsylvania, USA.

Received: 9 August 2012 Accepted: 19 September 2012 Published: 9 October 2012

\section{References}

1. Schroder AR, Shinn P, Chen H, Berry C, Ecker JR, Bushman F: HIV-1 integration in the human genome favors active genes and local hotspots. Cell 2002, 110:521-529.
2. Ciuffi A, Llano M, Poeschla E, Hoffmann C, Leipzig J, Shinn P, Ecker JR, Bushman F: A role for LEDGF/p75 in targeting HIV DNA integration. Nat Med 2005, 11:1287-1289

3. Shun MC, Raghavendra NK, Vandegraaff N, Daigle JE, Hughes S, Kellam P, Cherepanov P, Engelman A: LEDGF/p75 functions downstream from preintegration complex formation to effect gene-specific HIV-1 integration. Genes Dev 2007, 21:1767-1778.

4. Marshall HM, Ronen K, Berry C, Llano M, Sutherland H, Saenz D, Bickmore W, Poeschla E, Bushman FD: Role of PSIP1/LEDGF/p75 in lentiviral infectivity and integration targeting. PLOS One 2007, 2:e1340.

5. Gijsbers R, Ronen K, Vets S, Malani N, De Rijck J, McNeely M, Bushman FD, Debyser Z: LEDGF hybrids efficiently retarget lentiviral integration into heterochromatin. Mol Ther 2010, 18:552-560.

6. Gijsbers R, Vets S, De Rijck J, Ocwieja KE, Ronen K, Malani N, Bushman FD, Debyser Z: Role of the PWWP domain of lens epithelium-derived growth factor (LEDGF)/p75 cofactor in lentiviral integration targeting. J Biol Chem 2011, 286:41812-41825.

7. Schrijvers R, De Rijck J, Demeulemeester J, Adachi N, Vets S, Ronen K, Christ F, Bushman FD, Debyser Z, Gijsbers R: LEDGF/p75-Independent HIV-1 Replication Demonstrates a Role for HRP-2 and Remains Sensitive to Inhibition by LEDGINs. PLoS Pathog 2012, 8:e1002558.

8. Busschots K, Voet A, De Maeyer M, Rain JC, Emiliani S, Benarous R, Desender L, Debyser Z, Christ F: Identification of the LEDGF/p75 binding site in HIV-1 integrase. J Mol Biol 2007, 365:1480-1492.

9. Cherepanov P, Devroe E, Silver PA, Engelman A: Identification of an evolutionarily conserved domain in human lens epithelium-derived growth factor/transcriptional co-activator p75 (LEDGF/p75) that binds HIV-1 integrase. J Biol Chem 2004, 279:48883-48892.

10. Llano M, Vanegas M, Hutchins N, Thompson D, Delgado S, Poeschla EM: Identification and characterization of the chromatin-binding domains of the HIV-1 integrase interactor LEDGF/p75. J Mol Biol 2006, 360:760-773.

11. De Rijck J, Bartholomeeusen K, Ceulemans H, Debyser Z, Gijsbers R: High-resolution profiling of the LEDGF/p75 chromatin interaction in the ENCODE region. Nucleic Acids Res 2010, 38:6135-6147.

12. Ferris AL, Wu X, Hughes CM, Stewart C, Smith SJ, Milne TA, Wang GG, Shun $M C$, Allis CD, Engelman A, Hughes SH: Lens epithelium-derived growth factor fusion proteins redirect HIV-1 DNA integration. Proc Natl Acad Sci U S A 2010, 107:3135-3140.

13. Vandegraaff N, Devroe E, Turlure F, Silver PA, Engelman A: Biochemical and genetic analyses of integrase-interacting proteins lens epitheliumderived growth factor (LEDGF)/p75 and hepatoma-derived growth factor related protein 2 (HRP2) in preintegration complex function and HIV-1 replication. Virology 2006, 346:415-426.

14. Llano M, Saenz DT, Meehan A, Wongthida P, Peretz M, Walker WH, Teo W, Poeschla EM: An essential role for LEDGF/p75 in HIV integration. Science 2006, 314:461-464.

15. Vanegas M, Llano M, Delgado S, Thompson D, Peretz M, Poeschla E: Identification of the LEDGF/p75 HIV-1 integrase-interaction domain and NLS reveals NLS-independent chromatin tethering. J Cell Sci 2005, 118:1733-1743.

16. Zielske SP, Stevenson M: Modest but reproducible inhibition of human immunodeficiency virus type 1 infection in macrophages following LEDGFp75 silencing. J Virol 2006, 80:7275-7280.

17. ENCODE project consortium: The ENCODE (ENCyclopedia Of DNA Elements) Project. Science 2004, 306:636-640.

18. Barski A, Cuddapah S, Cui K, Roh TY, Schones DE, Wang Z, Wei G, Chepelev I, Zhao K: High-resolution profiling of histone methylations in the human genome. Cell 2007, 129:823-837.

19. Christ F, Voet A, Marchand A, Nicolet S, Desimmie BA, Marchand D, Bardiot D, Van der Veken NJ, Van Remoortel B, Strelkov SV, et al: Rational design of small-molecule inhibitors of the LEDGF/p75-integrase interaction and HIV replication. Nat Chem Biol 2010, 6:442-448.

20. Berry C, Hannenhalli S, Leipzig J, Bushman FD: Selection of target sites for mobile DNA integration in the human genome. PLoS Comput Bio/ 2006, 2:e157.

21. Schones DE, Cui K, Cuddapah S, Roh TY, Barski A, Wang Z, Wei G, Zhao K: Dynamic regulation of nucleosome positioning in the human genome. Cell 2008, 132:887-898

22. Madlala P, Gijsbers R, Christ F, Hombrouck A, Werner L, Mlisana K, An P, Karim SS, Winkler CA, Debyser Z, Ndung'u T: Association of Polymorphisms in the LEDGF/p75 Gene (PSIP1) with Susceptibility to HIV-1 Infection and Disease Progression. AIDS 2011, 25:1711-1719. 
23. Mous K, Jennes W, Camara M, Seydi M, Daneau G, Mboup S, Kestens L, Van Ostade X: Expression Analysis of LEDGF/p75, APOBEC3G, TRIM5alpha, and Tetherin in a Senegalese Cohort of HIV-1-Exposed Seronegative Individuals. PLoS One 2012, 7:e33934.

24. Vermeulen M, Eberl HC, Matarese F, Marks H, Denissov S, Butter F, Lee KK, Olsen JV, Hyman AA, Stunnenberg HG, Mann M: Quantitative interaction proteomics and genome-wide profiling of epigenetic histone marks and their readers. Cell 2010, 142:967-980.

25. Pradeepa MM, Sutherland HG, Ule J, Grimes GR, Bickmore WA: Psip1/Ledgf p52 Binds Methylated Histone H3K36 and Splicing Factors and Contributes to the Regulation of Alternative Splicing. PLoS Genet 2012, 8:e1002717.

doi:10.1186/1742-4690-9-84

Cite this article as: Schrijvers et al: HRP-2 determines HIV-1 integration site selection in LEDGF/p75 depleted cells. Retrovirology 2012 9:84.

\section{Submit your next manuscript to BioMed Central and take full advantage of:}

- Convenient online submission

- Thorough peer review

- No space constraints or color figure charges

- Immediate publication on acceptance

- Inclusion in PubMed, CAS, Scopus and Google Scholar

- Research which is freely available for redistribution 